\title{
STUDY AND ANALYSIS OF GRAPHS AND MODELS BASED INTERCONNECTION NETWORK SYSTEM
}

\author{
Raj Kumar Gupta ${ }^{1}$, Dr. K.L. Jaiswal ${ }^{2}$ \\ 1, 2 Model Science College, Rewa (M.P.), India \\ DOI: https://doi.org/10.29121/ijetmr.v8.i1.2021.872
}

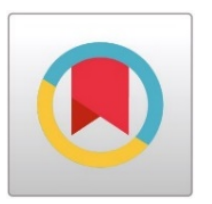

Article Citation: Raj Kumar Gupta, and Dr. K.L. Jaiswal. (2021). EFFICACY OF BASALT AND GRANITE AS COARSE AGGREGATE IN CONCRETE MIXTURE. International Journal of Engineering Technologies and Management Research, 8(1), 53-56. https://doi.org/10.29121/ijetmr.v8 i1.2021.872

Published Date: 31 January 2021

Keywords:

Interconnection Networks

Network Management

Fault Management

\begin{abstract}
The topology of network connections has become a vital step in the implementation of all conventional network networks. Cube architecture is one of the key aspects of network integration that explore and create networks of cubes. They praise and work together to complete all network management tasks. However, in this article, we will focus on the error handling function.
\end{abstract}

\section{INTRODUCTION}

These views are known as unpredictable networks, such as broadcasting marketing strategies, etc. Network connectivity plays an important role in determining how many systems are running. To address performance problems, studies such networks were performed with various types of multiprocessor systems. Thinking about real networks is a serious problem in the uniform design and distribution of computers. In general, a systematic search network using similar applications has no theoretical solution. There are several ways to get a work toy to be thrown away, which raises controversy over barriers to use.

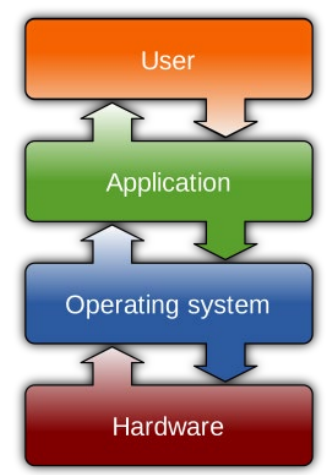

Figure 1: Flow diagram of user to hardware 


\section{PARAMETERS OF INTERCONNECTION NETWORK}

Metric numbers are often used to describe the function of a network connection.

\section{Degree or Connectivity}

The number of nodes (points) corresponds to this node. (This is the number of positions that can be obtained from this node in the course)

\section{Network Diameter}

The mesh diameter is the shortest path between two other nodes. Distance is measured as a number between two other nodes, the diameter should be small.

\section{Cost}

The cost of the network depends on the number of communication numbers required for the network.

\section{Average Node Distance}

The area between two areas is determined by the number of jumps on the small paths between them.

\section{Message Traffic Density}

\subsection{HYPERCUBE}

Hypercube is widely used as a component of systems such as Intel iPSC, nCUBE, which unites CM-2 and SGI Well 2000 machines. An n-sided hypercube or $n$ cube is $2 n$ nodes and has $n$ points on each. Nodes If only one n-bit binary address is assigned to an n-sided hypercube level, the link will connect two nodes, or and only if their binary addresses change at the same time.

\subsection{METACUBE}

The MC network consists of two cube-cube networks, which eliminates the problem of obstacles in the hypercube network, so that the number of sites in the network exceeds the number of points in a hypercube with a fixed number of connections on one. Node

\subsection{CROSSED CUBE}

$\mathrm{Cn}$, expressed as the n-dimensional cube of the crosstab, is obtained by navigating through other parts of the ndimensional hypercube. The crosstab cube has the same axis and edge as the Hypercube, but only half the width, largest diameter, and largest diameter. The crack looks like a hypercube of equal size.

\subsection{FOLDED CROSSED CUBE}

A fragmented cross section is created by connecting each node to the outermost node.

\subsection{STAR VERTICAL CUBE}

Star differentiation network cubes in which various hyperbolic patterns are included in the star graph.

\subsection{X-TORUS}

The X-torus network is a two-sided top. 


\subsection{STAR CROSSED CUBE}

$\operatorname{SCC}(\mathrm{k}, \mathrm{m})$ is a multigraph CC $(\mathrm{m})$ and an n-sided star graph S (n).

\section{COMPARATIVE STUDY OF VARIOUS ARCHITECTURE}

Node, diameter level and mean between locations, messaging rates across buildings versus HC, MC, CC, FCC, SVC, X-TORUS, SCC and SMC locations. We are still looking at the parameter. There are four key factors such as cpu frequency, diameter, average, and message traffic rate.

\section{FAULT MANAGEMENT}

In this section, we define some terms related to network outages and highlight some of the common problems that arise when you fail.

\subsection{DEFINITION OF TERMS AND FAULT CHARACTERISTICS}

The words "wrong", "wrong" and "wrong" are often misunderstood. Misconceptions about these terms may lead to misuse. A detailed explanation can be found in Wang's article. Defective software or hardware load on the system that degrades communication or makes characters degrade. The invalid error does not come from a system segment. If the product is defective, we would like to say that it cannot be used. This does not work

The failure or failure of an object depends on the failure of the object. It is important to distinguish between the terms described above. This is a direct or indirect mistake. Errors are indicative of inefficiency.

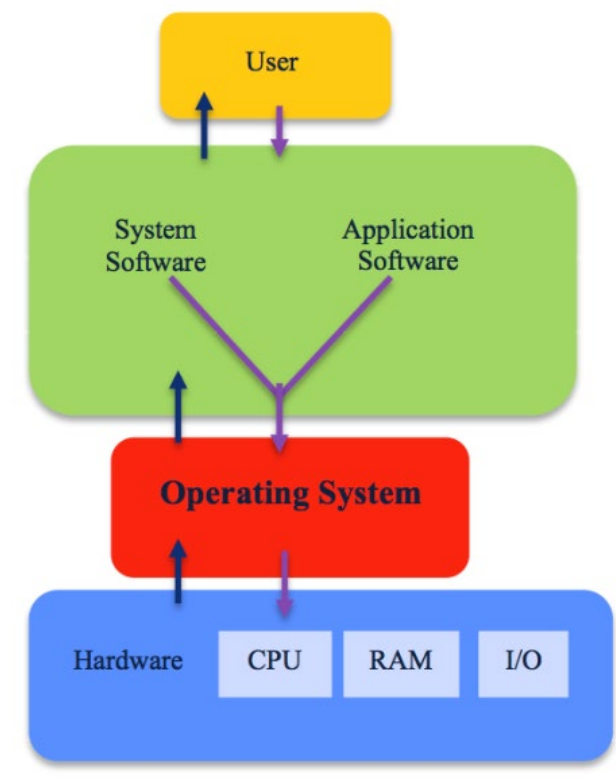

Figure 2: Fault management of computer system

\subsection{FAULT MANAGEMENT PROCESS}

Full error handling system including auditing, reporting, error saving logging, filtering, compatibility, testing and recovery. This section seeks to talk about areas where management will fail. For the purposes of this discussion, we have decided to break down the error correction process into four main categories: search, isolation, correction and. Management To solve the problem described above, we created and installed FMS. There are three types of error handling applications as shown. It meant the hassle of torture, recording, and diagnostic testing. 


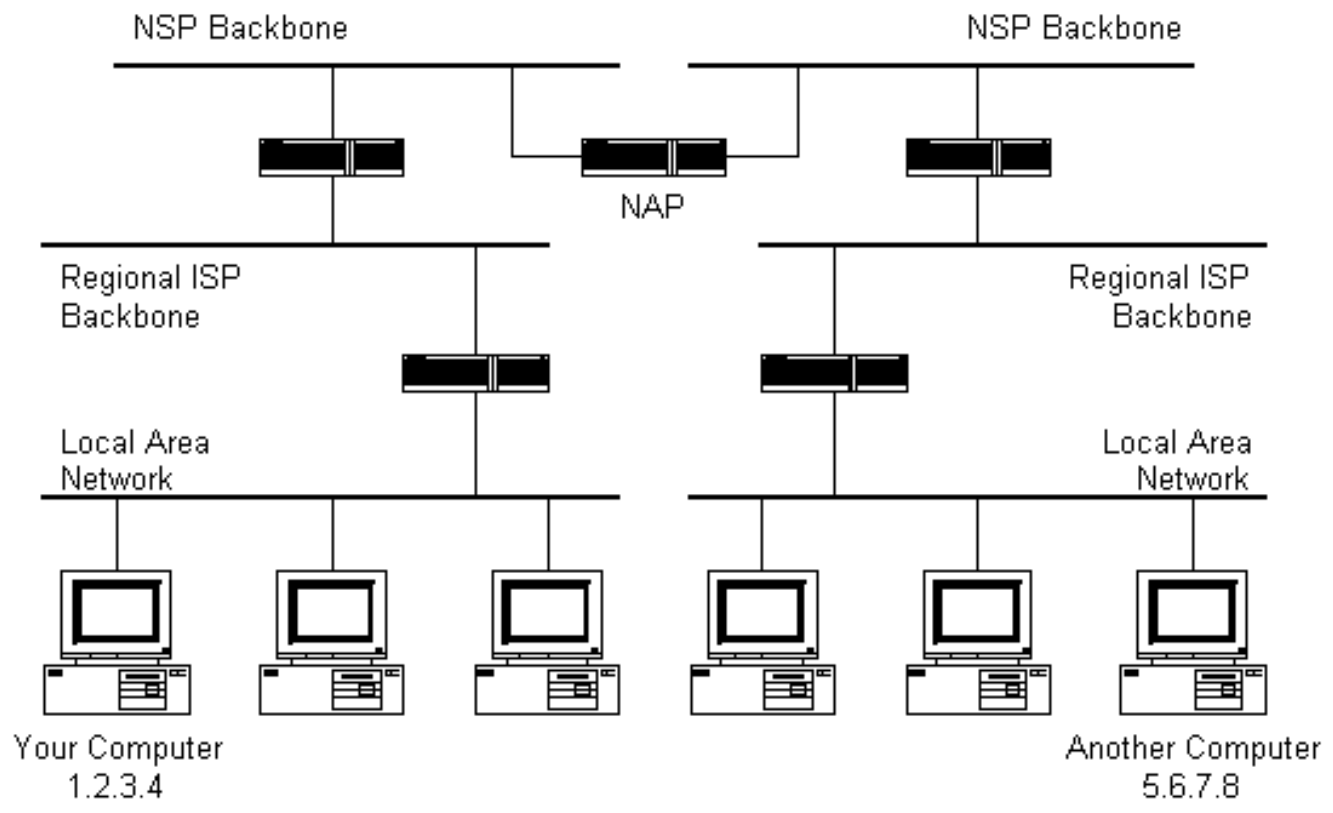

Figure 3: Fault Management Process of computer system

\section{CONCLUSIONS}

There are studies comparing different types of relations with the National Assembly. Nowadays, most people rely on computers to work. This means that the computer network must be available and provide the best service in most cases. Failure to be exact and incompatible is unacceptable and must be prevented. Therefore, promoting network failure management is an important factor to consider when planning a computer network.

\section{SOURCES OF FUNDING}

This research received no specific grant from any funding agency in the public, commercial, or not-for-profit sectors.

\section{CONFLICT OF INTEREST}

The author have declared that no competing interests exist.

\section{ACKNOWLEDGMENT}

None.

\section{REFERENCES}

[1] A. Agarwal, "Exploring Alternative Topologies for Network-on-Chip Architectures," BIJIT - BVICAM's International Journal of Information Technology Vol. 3, No. 2, ISSN 0973 - 5658, 2011

[2] N. K. Swain and N. Adhikari, "Star varietal cube: A New Large Scale Parallel Interconnection Network", International Journal Communication \& Network Security (IJCNS), Vol. 1, Issue-II, 2011.

[3] J. Zhang, and Y. Li "X-Torus: A Variation of Torus Topology with Lower Diameter and Larger Bisection Width".

[4] G. Tao, "Xtorus: An Extended Torus Topology for On-Chip Massive Data Communication," 26th IEEE Int. Parallel and Distributed Processing Symposium Workshops \& PhD Forum (IPDPSW), pp. 2061-2068, 2012.

[5] Wang, "Model of Network Faults", Integrated Network Management I, B. Meandzija and J. Westcott (Editors) Elsevier Science Publishers B.V. (North-Holland), IFIP, 1989. 